\title{
Syndrome de Gardner diagnostiqué à partir de manifestations dento-maxillaires
}

\author{
Adel Ayat ${ }^{1,{ }^{*}}$, Karim Layaida ${ }^{2}$, Badia Saari ${ }^{3}$, Zahia Boudaoud ${ }^{3}$ \\ ${ }^{1}$ Service de Pathologie et Chirurgie buccale, Hôpital militaire régional universitaire, Constantine, Algérie \\ 2 Service de Gastroentérologie, CHU Mustapha, Alger, Algérie \\ ${ }^{3}$ Service de Pathologie et Chirurgie buccale, CHU Mustapha, Algérie
}

(Reçu le 14 avril 2013, accepté le 12 mai 2013)

Mots clés : syndrome de Gardner / polypose adénomateuse familiale / ostéome / dents incluses
Key words:

Gardner's syndrome /

familial adenomatous polyposis /osteoma / impacted teeth
Résumé - Le syndrome de Gardner, variante phénotypique de la polypose adénomateuse familiale (PAF), se caractérise par la triade suivante : une polypose colorectale, des fibromes desmoides abdominaux ou cutanés et des lésions dento-maxillaires très polymorphes, faites d'ostéomes, d'odontomes, de dents surnuméraires et de dents incluses. Le problème majeur de cette maladie, à transmission autosomique dominante, réside dans les polypes qui se transforment en adénocarcinomes à partir de la quatrième décennie. Le chirurgien dentiste peut être amené à évoquer le syndrome et à jouer un rôle dans le dépistage précoce puisque les manifestations dento-maxillaires observées dès le jeune âge précèdent les lésions digestives. L'objectif de ce travail est de relater l'observation d'une adolescente de 15 ans dont le tableau clinique a incité à rechercher un syndrome de Gardner. Le diagnostic confirmé par les explorations gastro-entérologiques a ainsi permis d'étendre les investigations à toute la famille et de détecter de nombreux membres atteints.

\begin{abstract}
Gardner's syndrome: dental maxillary features contributing to the diagnosis. Gardner's syndrome, phenotypic variant of the familial adenomatous polyposis (FAP), is characterized by the triad: colorectal polyposis, desmoid fibromas and sebaceous cysts and dento-maxillary lesions made of highly polymorphic, such as osteomas, odontomas, supernumerary teeth and impacted teeth. The major problem of this disease with dominant autosomal transmission lies in the polyps which are then transformed into adenocarcinomas from the fourth decade. The dental surgeon can be brought to evoke the syndrome and to play a role in the early detection. This role is due to dental lesions that can be observed from the young age and precede the gastrointestinal damage. The purpose of this work is to relate a case report of 15 year-old girl whose clinical picture prompted us to search for the Gardner syndrome. The diagnosis was confirmed by gastrointestinal examination. The case control also allowed to spread the investigations to all the family and to detect numerous affected members.
\end{abstract}

Le syndrome de Gardner est une affection génétique rare, à transmission autosomique dominante. Il doit son nom à l'auteur qui, en 1951, a décrit le syndrome en y ajoutant les anomalies dentaires et en insistant sur l'importance des anomalies bucco-dentaires pour le diagnostic.

Le colon et le rectum des sujets atteints sont le siège de très nombreux (quelquefois plus d'une centaine) polypes adénomateux, qui apparaissent le plus souvent vers la deuxième ou la troisième décade [1]. En l'absence de traitement chirurgical prophylactique, ces polypes évoluent immanquablement vers l'apparition d'un ou de plusieurs cancers coliques ou rectaux, généralement après l'âge de 40 ans.
La polypose adénomateuse familiale (PAF) représente une entité comportant un phénotype variable avec des signes coliques et éventuellement extra-digestifs [2]. Elle est due à une mutation du gène APC (adenomatous polyposis coli) situé sur le bras long du chromosome 5. Le syndrome de Gardner est une variante phénotypique de la PAF. Les sujets affectés présentent une PAF associée à différents symptômes : des tumeurs desmoides gastriques, des kystes sébacés ou épidermoïdes, une hypertrophie congénitale de l'épithélium pigmentaire de la rétine, une pathologie dento-maxillaire variée...

Les manifestations dento-maxillaires cliniques et radiologiques observées chez une patiente de 15 ans, ont conduit

\footnotetext{
*Correspondance : adel.rida@gmail.com
} 
à solliciter l'avis d'un gastroentérologue. Le diagnostic de syndrome de Gardner a été posé sur un faisceau de signes digestifs et odonto-stomatologiques. Dans un deuxième temps, le diagnostic a été porté chez plusieurs membres de la famille à la suite d'une enquête.

\section{Observation}

Une patiente âgée de 15 ans, diabétique type I, a été adressée pour une tuméfaction maxillaire gauche évoluant depuis deux mois. Cette tuméfaction vestibulaire, étendue de la 22 à la 26, était recouverte par une muqueuse d'aspect bleuté. Les dents $13,14,23$ et 24 étaient absentes et il y avait une persistance de la 82 (Fig. 1).

La radiographie panoramique des maxillaires a mis en évidence plusieurs anomalies (Fig. 2). Au maxillaire, on notait la présence d'une image kystique d'environ $6 \mathrm{~cm}$ de diamètre s'entendant de la 21 à la 27 , englobant 23 et 24 en position haute juxta-sinusienne. La 13 était refoulée prés de la paroi latérale des fosses nasales, tandis que la 14 était distalée au-dessus des racines de la 26. L'ostéolyse était centrée sur deux lésions radio-opaques, d'environ $0,5 \mathrm{~cm}$ et $1 \mathrm{~cm}$ de diamètre. On notait également la présence d'une lésion radioopaque entre le tiers apical des racines de 21 et de 22, entourée d'un halo radio-clair. À la mandibule, il existait trois formations radio-opaques: l'une au-dessus de la 38 faisant évoquer un odontome, l'autre de $1 \mathrm{~cm}$ de diamètre près de la racine de la 35 plutôt un ostéome et la troisième d'environ $0,5 \mathrm{~cm}$ de diamètre un odontome situé en position coronaire par rapport à la 42 retenue. L'examen tomodensitométrique a mis en évidence la présence d'une lésion radiotransparente maxillaire gauche de $5,5 \mathrm{~cm}$ dans son grand axe, englobant deux dents refoulées près du sinus maxillaire et deux images radio-opaques, de tonalité dentaire dont la plus volumineuse avait $1,5 \mathrm{~cm}$ de diamètre. Cette image faisait évoquer un kyste dentigère renfermant plusieurs dents et du matériel minéralisé. L'examen tomodensitométrique mettait aussi en évidence deux images radio-opaques de $0,5 \mathrm{~cm}$ de diamètre dans la région rétro-incisive maxillaire gauche (Fig. 3 ).

Ce tableau radiologique regroupant odontomes, inclusions dentaires multiples, ostéome et formation kystique a fait évoquer un syndrome de Gardner. Ce diagnostic a été conforté par les antécédents familiaux qui orientaient vers une probable PAF au regard des interventions chirurgicales abdominales subies par le père et deux tantes.

La coloscopie réalisée chez notre patiente a objectivé la présence de plusieurs polypes coliques et rectaux (Fig. 4). La biopsie du plus volumineux (2 $\mathrm{cm}$ de diamétre) ne montrait aucun signe de dysplasie. L'examen ophtalmologique pour rechercher une hypertrophie congénitale de l'épithélium pigmentaire de la rétine a révélé un glaucome de l'œil droit. L'examen dermatologique était sans particularités.

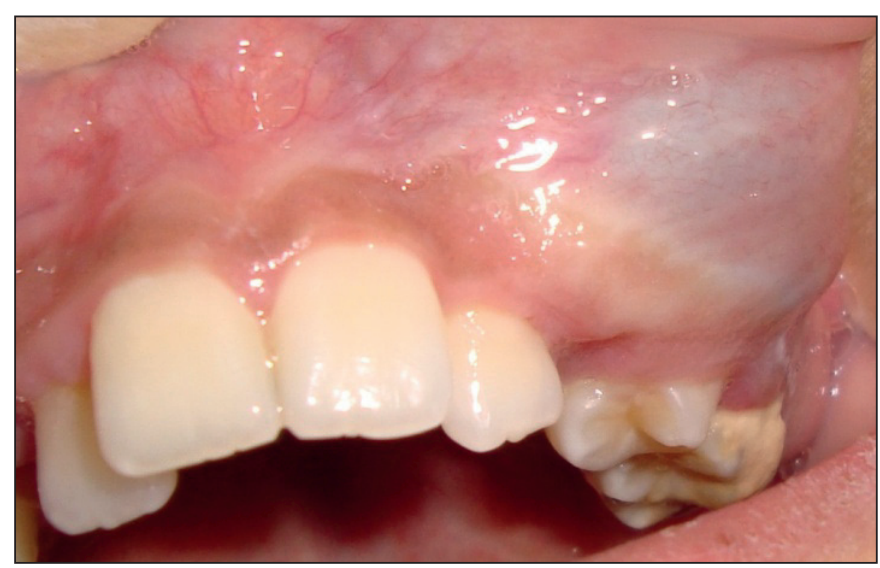

Fig. 1. Vue endobuccale : tuméfaction vestibulaire du maxillaire gauche.

Fig. 1. Intraoral view: vestibular swelling of the left maxillary.

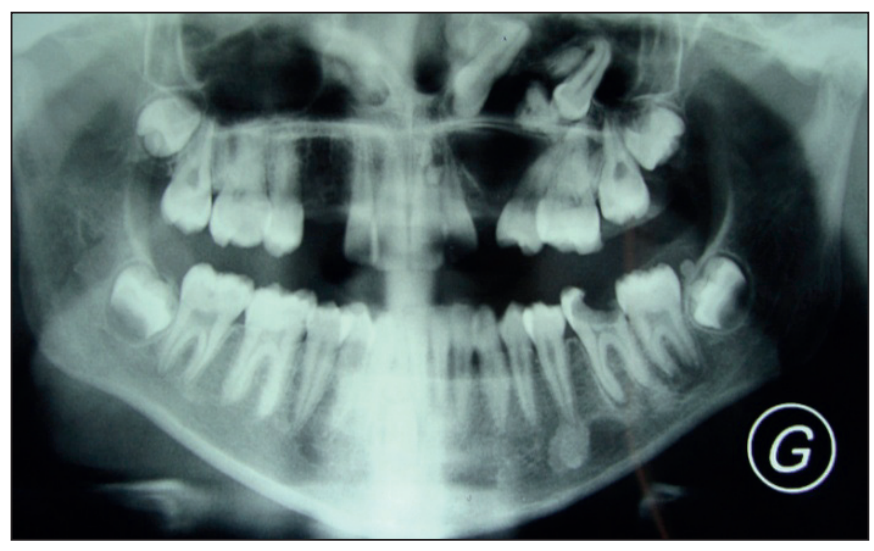

Fig. 2. Radiographie panoramique : anomalies dento-maxillaires. Fig. 2. Panoramic radiograph: dento-maxillary abnormalities.

L'énucléation kystique emportant les 23 et 24 a été réalisée sous anesthésie locorégionale. L'examen histopatholologique a montré que la lésion kystique comportait une paroi conjonctive fibro-vasculaire tapissée d'un épithélium pluristratifié rappelant l'épithélium adamantin. Au sein de cet épithélium, on observait des masses constituées de cellules momifiées ou cellules fantômes. Par endroits, des zones pseudo-améloblastiques semblaient infiltrer la paroi kystique. Cet aspect était compatible avec un kyste odontogène calcifiant (Fig. 5).

L'ensemble de ces éléments a conduit au diagnostic du syndrome de Gardner. Une surveillance coloscopique biannuelle a été instaurée chez cette patiente. Devant le caractère héréditaire du syndrome de Gardner, une enquête familiale a été déclenchée. L'entretien avec les membres de la famille accompagnant la patiente a été fait en vue de les informer et de les sensibiliser sur les risques que comporte la maladie. Une radiographie panoramique et un examen coloscopique ont été programmés chez tous les membres de la famille acceptant de se faire examiner. Les résultats de l'enquête familiale sont 


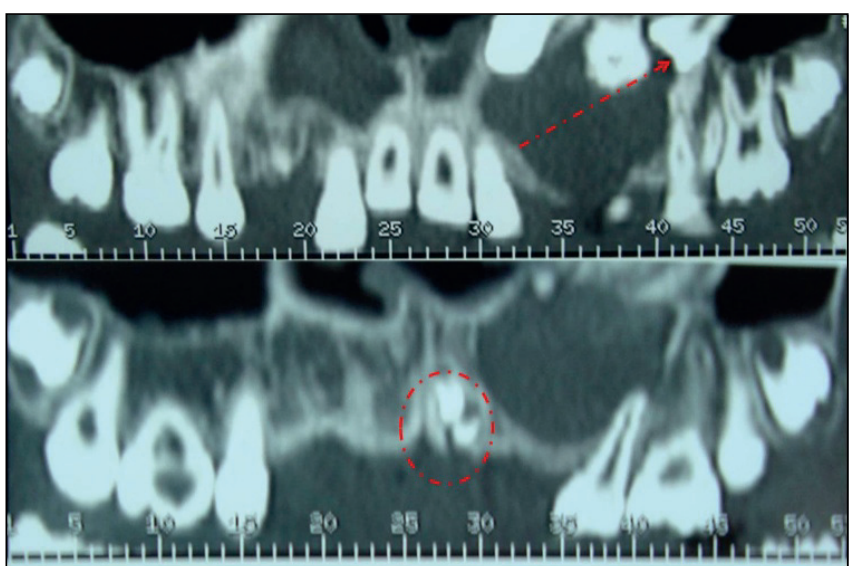

Fig. 3. Coupes tomodensitométriques du maxillaire. Fig. 3. Computed tomography slices of the maxillary.

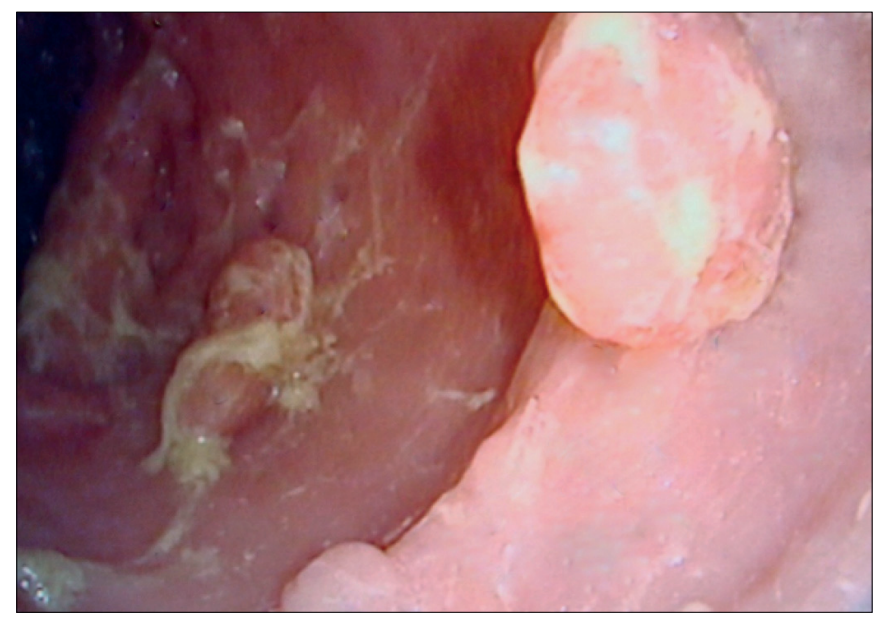

Fig. 4. Polypose colique.

Fig. 4. Colonic polyposis.

mentionnés sur l'arbre généalogique (Fig. 6) et dans le tableau récapitulatif des pathologies retrouvées (Tab. I).

\section{Commentaires}

Le syndrome de Gardner est caractérisé par une triade comportant des polypes colorectaux qui présentent fréquemment une transformation maligne, des tumeurs des tissus mous et des tumeurs osseuses qui intéressent principalement mais non exclusivement les os de la face et du crâne. La maladie, due à une mutation du gène $A P C$, est autosomique dominante avec une pénétrance complète et une expressivité variable.

Les signes extra-digestifs précèdent de plusieurs années la polypose colique souvent asymptomatique [3]. Il est important de sensibiliser les chirurgiens-dentistes à ces signes, à leur signification notamment chez l'enfant ou l'adolescent. La symptomatologie dento-maxillaire très riche, retrouvée chez cette jeune patiente, comportant des inclusions dentaires multiples,

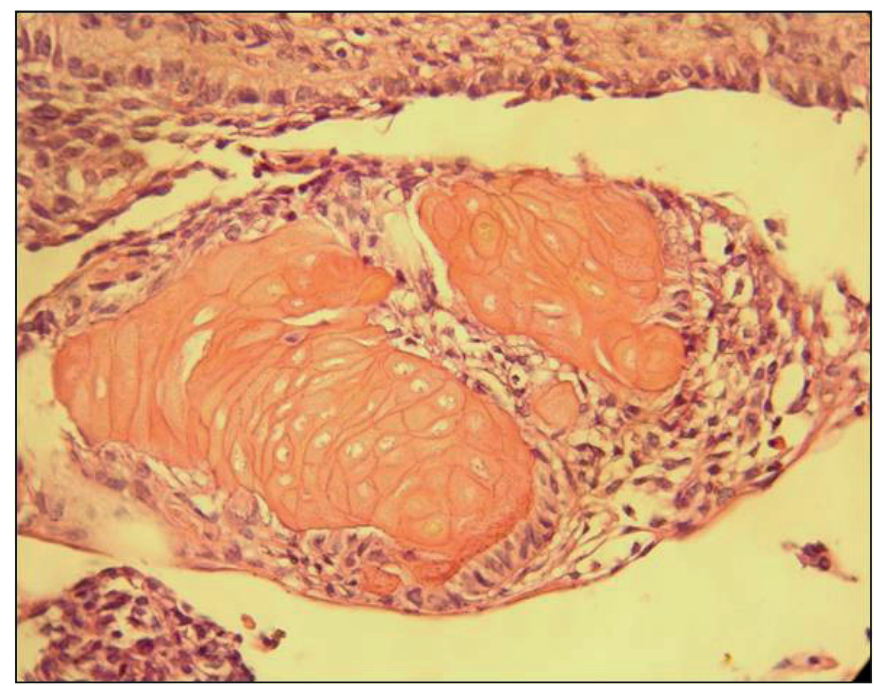

Fig. 5. Aspect histologique du kyste odontogène calcifiant : amas de cellules fantômes.

Fig. 5. Histopathological appearance of the calcifying odontogenic cyst: pile of ghost cells.

plusieurs odontomes, un ostéome et une lésion radioclaire inhomogène, a fait suspecter et rechercher une atteinte syndromique. Les antécédents familiaux - le père de la patiente et deux de ses tantes sont décédés d'un cancer colorectal ; ces dernières à l'âge de 40 et 49 ans - ont contribué à confirmer le diagnostic de syndrome de Gardner. Dans la plupart des cas de syndrome de Gardner publiés dans la littérature odontologique, on décrit plutôt les manifestations dento-maxillo-faciales d'un syndrome déjà connu [4-6]. Plus rarement, ce sont les manifestations dento-maxillaires qui ont conduit à la découverte d'un syndrome méconnu $[7,8]$.

L'âge moyen lors du diagnostic est d'environ 32 ans dans la série rapportée. Un diagnostic plus précoce devrait être possible, compte tenu de la présence des manifestations stomatologiques dès l'enfance ou l'adolescence [9]. Les différents examens digestifs endoscopiques ont révélé des anomalies significatives. La polypose colique est un élément quasi constant, retrouvé dans 19 cas sur 21 , soit de $90 \%$ des sujets (Tab. I). Ce taux est comparable à celui de la littérature $[9,10]$. Les polypes adénomateux sont souvent présents dès l'adolescence et précèdent d'environ deux décennies la dysplasie et le cancer ; ceci explique le nombre inférieur de dysplasies ou de cancers chez nos patients. Les dysplasies incitent à la surveillance biannuelle par coloscopies [11, 12].

L'hypertrophie congénitale de l'épithélium pigmentaire de la rétine, signe ophtalmologique associé dans $75 \%$ des cas, était absente dans le cas rapporté mais cette jeune fille présentait un glaucome.

Elle était également atteinte d'un diabète de type I. Cette affection fait partie des désordres endocriniens retrouvés dans 


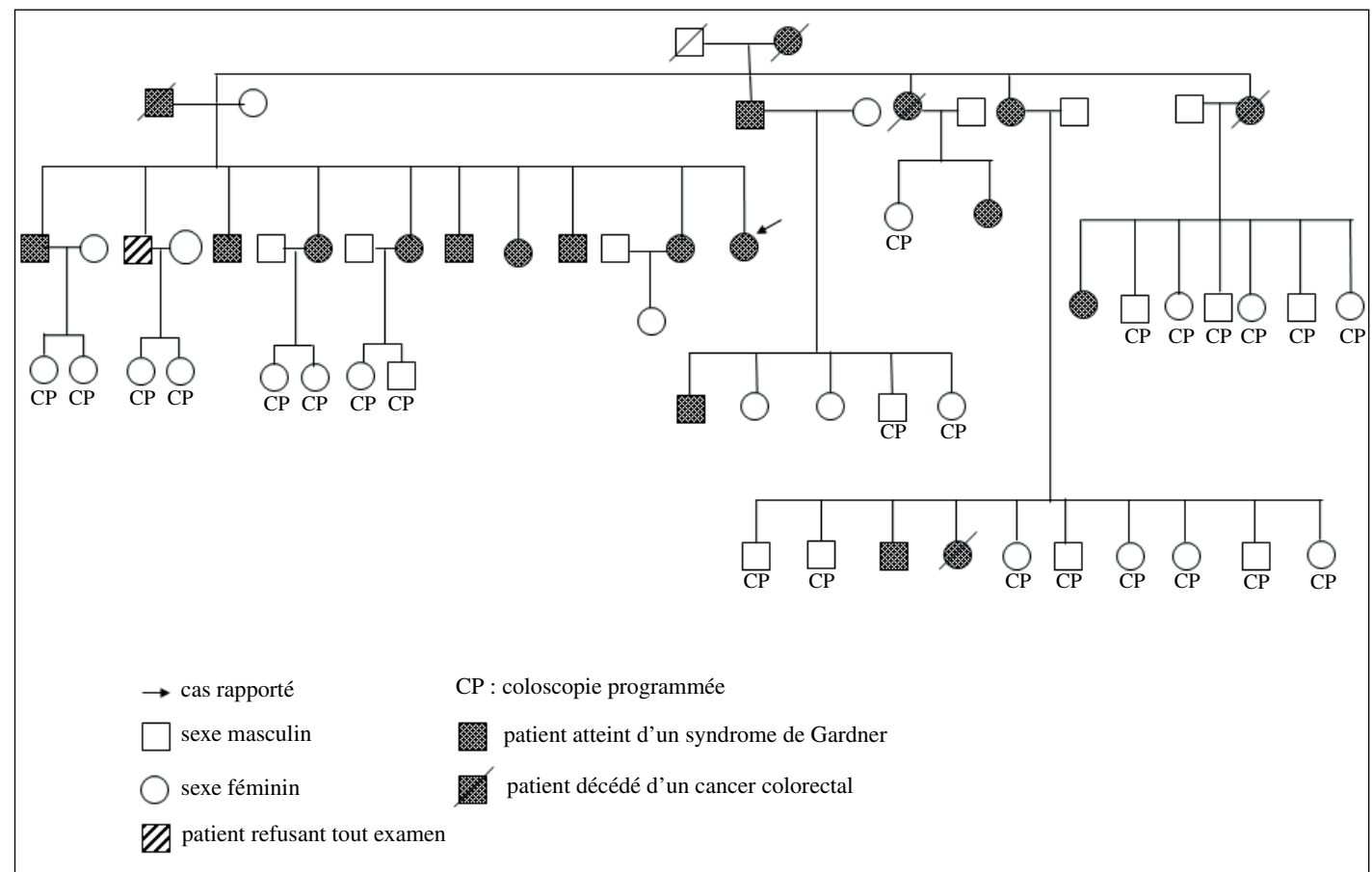

Fig. 6. Arbre généalogique de la famille du cas rapporté.

Fig. 6. Family genealogical tree of the reported case.

Tableau I. Tableau récapitulatif des lésions observées lors de l'examen stomatologique et radiologique et lors des colonoscopies. Table I. Summary table of observed lesions during stomatological and radiological examination and during colonoscopies.

\begin{tabular}{|c|c|c|c|c|c|c|c|c|c|}
\hline \multirow{2}{*}{$\begin{array}{l}\text { Membres } \\
\text { examinés }\end{array}$} & \multirow{2}{*}{$\begin{array}{c}\text { Âge } \\
\text { (années) }\end{array}$} & \multirow{2}{*}{ Sexe } & \multicolumn{4}{|c|}{ Lésions dento-maxillaires } & \multicolumn{3}{|c|}{ Lésions colorectales } \\
\hline & & & dents sumuméraires & odontome & inclusion & ostéomes & polypes & dysplasies & cancer \\
\hline 1 & 61 & $\hat{\sigma}$ & - & - & - & + & + & + & + \\
\hline 2 & 35 & $\hat{\sigma}$ & - & + & + & - & + & + & - \\
\hline 3 & 31 & $\hat{0}$ & + & + & + & + & + & + & - \\
\hline 4 & 29 & q & - & - & + & + & + & + & - \\
\hline 5 & 27 & $\hat{o}$ & - & + & + & + & + & + & - \\
\hline 6 & 26 & P & - & - & - & + & + & + & - \\
\hline 7 & 24 & q & - & - & + & + & + & - & - \\
\hline 8 & 22 & P & - & - & - & - & + & + & - \\
\hline 9 & 20 & $\hat{0}$ & + & + & + & - & + & - & - \\
\hline$\underline{10}$ & 15 & q & - & + & + & + & + & - & - \\
\hline 11 & 53 & $\pi$ & $\mathrm{NE}$ & $\mathrm{NE}$ & $\mathrm{NE}$ & $\mathrm{NE}$ & + & + & + \\
\hline 12 & 22 & $\pi$ & - & + & + & - & + & + & - \\
\hline 13 & 16 & $\hat{0}$ & - & - & - & + & $\mathrm{NE}$ & $\mathrm{NE}$ & $\mathrm{NE}$ \\
\hline 14 & 13 & ㅇ & - & + & + & - & $\mathrm{NE}$ & $\mathrm{NE}$ & $\mathrm{NE}$ \\
\hline 15 & 54 & ㅇ & $\mathrm{NE}$ & $\mathrm{NE}$ & $\mathrm{NE}$ & $\mathrm{NE}$ & + & + & - \\
\hline 16 & 34 & $\pi$ & $\mathrm{NE}$ & $\mathrm{NE}$ & $\mathrm{NE}$ & $\mathrm{NE}$ & + & + & - \\
\hline 17 & 32 & q & $\mathrm{NE}$ & $\mathrm{NE}$ & $\mathrm{NE}$ & $\mathrm{NE}$ & + & + & + \\
\hline 18 & 40 & q & $\mathrm{NE}$ & $\mathrm{NE}$ & $\mathrm{NE}$ & $\mathrm{NE}$ & + & + & + \\
\hline 19 & 25 & P & $\mathrm{NE}$ & $\mathrm{NE}$ & $\mathrm{NE}$ & $\mathrm{NE}$ & + & - & - \\
\hline 20 & 49 & q & $\mathrm{NE}$ & $\mathrm{NE}$ & $\mathrm{NE}$ & $\mathrm{NE}$ & + & + & + \\
\hline \multirow[t]{2}{*}{21} & 35 & q & $\mathrm{NE}$ & $\mathrm{NE}$ & $\mathrm{NE}$ & $\mathrm{NE}$ & + & + & - \\
\hline & moy $=32$ & $9 \delta^{\wedge} 12$ P & $2 / 13$ & $7 / 13$ & $9 / 13$ & $8 / 13$ & 19 & 15 & 5 \\
\hline
\end{tabular}

NE : Non Examiné 
le syndrome de Gardner [13] où l'on peut également rencontrer des néoplasies endocriniennes. Le carcinome papillaire de la thyroïde représente la néoplasie la plus commune chez ces patients et nécessite un suivi régulier.

Les lésions osseuses sont dominées par les ostéomes. Ces tumeurs bénignes de localisation presque exclusivement maxillo-faciale, concernent tous les os de la face, les sinus paranasaux mais surtout la mandibule [14]. Les ostéomes peuvent être uniques ou multiples se traduisant par de petites opacités bien limitées, localisées surtout dans la portion alvéolaire, formant des énostoses ou des exostoses. Ils sont à différencier des odontomes ou des cémentomes car ces deux entités font également partie du syndrome. Les formations périphériques (exostoses) siègent le plus souvent sur le rebord basilaire où ils forment des saillies palpables ou visibles lorsqu'elles refoulent le revêtement cutané [15]. Les ostéomes sont présents chez 68 à $82 \%$ des patients alors que la prévalence des ostéomes dans la population générale est inférieure à $10 \%[16,17]$. Le cas rapporté présentait un ostéome mandibulaire d'évolution lente, asymptomatique. Dans la série rapportée, 8 patients sur 14 présentaient un ou plusieurs ostéomes. Sur le plan thérapeutique, malgré leur tendance à la récidive [4], les ostéomes peuvent être justiciables d'une résection chirurgicale. Cependant, lorsqu'ils sont asymptomatiques, surtout pour les énostoses dont l'exérèse peut entrainer un délabrement osseux important, il n'y a aucune indication pour intervenir [18].

Les anomalies dentaires sont observées dans $70 \%$ des cas, alors que seulement $1 \%$ de la population générale présente des anomalies dentaires [19]. Elles comprennent des agénésies dentaires, des dents surnuméraires, des inclusions multiples, des kystes dentigères, des odontomes, des hypercémentoses, des microdonties. Ces anomalies représentent l'élément le plus constant et il a été retrouvé chez 12 des 13 patients explorés. L'incidence des dents surnuméraires dans la population générale est de 0,1 à 3,6\% alors que qu'elle est de $38 \%$ dans le syndrome de Gardner [20]. Les syndromes les plus connus qui s'accompagnent de dents surnuméraires sont le syndrome de Gardner et le syndrome de Pierre Marie et Sainton ou dysostose cleido-cranienne.

Enfin, la patiente présentait une tumeur maxillaire d'allure kystique radiologiquement inhomogène. L'analyse histopathologique a montré qu'il s'agissait d'un kyste odontogène calcifiant. Ce kyste n'a jamais été décrit dans le syndrome de Gardner.

\section{Conclusion}

Le chirurgien dentiste doit être particulièrement attentif lorsqu'il découvre plusieurs anomalies bucco-dentaires comme la présence d'ostéomes, d'anomalies dentaires et d'odontomes, chez un même patient. Ces anomalies accessibles à l'examen clinique et décelables avec une simple radiographie panoramique peuvent orienter vers le diagnostic de PAF, surtout si l'histoire anamnestique est très évocatrice. Le chirurgien dentiste peut donc participer au dépistage d'un cancer colorectal dont le traitement chirurgical précoce par colectomie préventive donne des résultats satisfaisants.

\section{Conflits d'intérêt : aucun}

\section{Références}

1. Aretz S. The differential diagnosis and surveillance of hereditary gastrointestinal polyposis syndromes. Dtsch Arztebl Int 2010;107:163-73.

2. Allen BA, Terdiman JP. Hereditary polyposis syndromes and hereditary non-polyposis colorectal cancer. Best Pract Res Clin Gastroenterol 2003;17:237-58.

3. Chung J, Namkoong S, Jung KE, Park JW, Park BC, Cinn YW, Kim MH. A case of Gardner's syndrome associated with desmoid tumor. Ann Dermatol 2010;22:418-21.

4. Touré G. Intérêts des signes maxillo-faciaux dans le diagnostic du syndrome de Gardner. Rev Stomatol Chir Maxillofac 2004; 105:177-81.

5. Ben Lagha N, Galéazzi J-M, Oxeda P, Bouhnik Y, Maman L. Syndrome de Gardner avec traitement chirurgical des ostéomes multiples. À propos d'un cas. Med Buccale Chir Buccale 2003;9:177-84

6. Chimenos-Küstner E, Pascual M, Blanco I, Finestres F. Hereditary familial polyposis and Gardner's syndrome: contribution of the odonto-stomatology examination in its diagnosis and a case description. Med Oral Patol Oral Cir Bucal 2005;10:402-9.

7. Herford AS, Stoffella E, Tandon R. Osteomas involving the facial skeleton: a report of 2 cases and review of the literature. Oral Surg Oral Med Oral Pathol Oral Radiol Endod 2013;115:e1-6.

8. Lee BD, Lee W, Oh SH, Min SK, Kim EC. A case report of Gardner syndrome with hereditary widespread osteomatous jaw lesions. Oral Surg Oral Med Oral Pathol Oral Radiol Endod 2009;107: e68-72.

9. Oner AY, Pocan S. Gardner's syndrome: a case report. Br Dent J 2006;200:666-7.

10. Basarana G, Erkanb M. One of the rarest syndromes in dentistry: Gardner syndrome. Eur J Dent 2008;2:208-12.

11. Wijn MA, Keller JJ, Giardiello FM, Brand HS. Oral and maxillofacial manifestations of familial adenomatous polyposis. Oral Dis 2007;13:360-5.

12. Jasperson KW, Tuohy TM, Neklason DW, Burt RW. Hereditary and familial colon cancer. Gastroenterology 2010;138:2044-58.

13. Mejdoub Rekik N, Ben Salah S, Kallel N, Kamoun M, Charfi N, Abid M. Adrenocortical secreting mass in a patient with Gardner's syndrome: a case report. Case Report Med 2010, $4 \mathrm{p}$.

14. Brkic A, Gürkan-Köseoğlu B, Özçamur Ç. Oral and maxillofacial manifestations of Gardner's syndrome: case report. Acta Stomatol Croat 2009;43:60-5.

15. Fonseca LC, Kodama NK, Nunes FC, Maciel PH, Fonseca FA, Roitberg M, de Oliveira JX, Cavalcanti MG. Radiographic 
assessment of Gardner's syndrome. Dentomaxillofac Radiol 2007;36:121-4.

16. Kamel SG, Kau CH, Wong ME, Kennedy JW, English JD. The role of Cone beam CT in the evaluation and management of a family with Gardner's syndrome. J Craniomaxillofac Surg 2009;37: 461-8.

17. Cankaya AB, Erdem MA, Isler SC, Cifter M, Olgac V, Kasapoglu C, Oral CK. Oral and maxillofacial considerations in Gardner's syndrome. Int J Med Sci 2012;9:137-41.
18. Madani M, Madani F. Gardner's syndrome presenting with dental complaints. Arch Iranian Med 2007;10:535-9.

19. Karazivan M, Manoukian K, Lalonde B. La polypose adénomateuse familiale ou syndrome de Gardner. Revue de la littérature et présentation de deux cas cliniques. J Can Dent Assoc 2000;66: 26-30.

20. Rajab LD, Hamdan MA. Supernumerary teeth: review of the literature and a survey of 152 cases. Int J Peadiatr Dent 2002; 12:244-54. 